\section{Tolerance Flux of Thermal Neutrons}

THr danger of neutrons on the human body arises from two factors : the slowing down of fast neutrons and the absorption of neutrons. In the case of thermal neutrons, only absorption can take place, either in hydrogen to form a deuteron with emission of a $2 \cdot 2-\mathrm{MeV} . \gamma$-ray, or by nitrogen-14 to form a carbon, with the emission of a $0 \cdot 7-\mathrm{MeV}$. proton. In a very complete paper on the subject, Mitchell ${ }^{1}$ finds that the ionization produced in the human body can be attributed half to the $\gamma$-rays of the latter reaction and half to the protons. This note deals only with the $\gamma$-rays from the $H(n, \gamma) D$ reaction.

Mitchell starts from the idea that the distribution of neutrons in the body is given by an exponential function, and he works out the ionization produced at the surface by $\gamma$-rays liberated in the semi-infinite medium. With a diffusion length of $2.8 \mathrm{~cm}$. he finds that the maximal dose is attained with an incident flux of thermal neutrons of the order of 1,000-2,000 neutrons/cm. $2 / \mathrm{sec}$.

It is well known that the ordinary theory of diffusion of thermal neutrons cannot be applied near a discontinuity (the surface of the body in this case), and as we expected that a purely exponential function would $b$ invalid and could vitiate the result quite a lot, we have set up an experiment to determine the true distribution function with a thermal neutron source outside $\approx$ paraffin block. This source was a 2,400-mc. radium-beryllium source in a howitzer from which neutrons are 'focused' on to a paraffin block $50 \mathrm{~cm}$. away. The detectors were rhodium plates $0.05 \mathrm{gm} . / \mathrm{cm} .{ }^{2}$ irradiated for five minutes, the activities of which were measured on both sides of the detector. Those detectors were irradiated at different depths inside and outside the paraffin and in such conditions that the contribution from the different kinds of neutrons could be determined. The results are shown in Fig. 1. Curve $A$ gives the total activity of the rhodium; curve $B$ gives the activity when a large cadmium screen completely shielded the paraffin block; this activity being due, therefore, to the resonance neutrons and the thermal neutron byproducts of the resonance slowed down to thermal energies. Curve $C$, with the rhodium plates completely shielded in a cadmium envelope, gives the distribution of resonance neutrons. Curve $E$ (equals $A-B$ ) gives the distribution of thermal neutrons only.

Activities measured at the surface and outside the paraffin cannot be attributed to neutrons travelling in the direction howitzer-paraffin only, but also to neutrons scattered and diffusing back in the direction paraffin-howitzer. It can be calculated that, in our

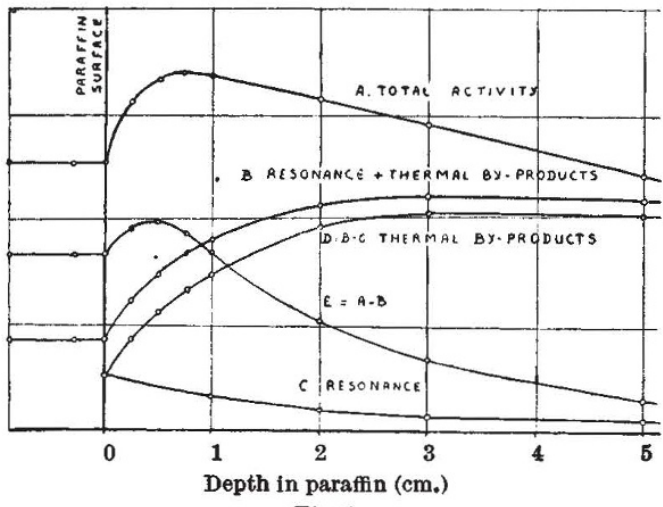

Fig. 1

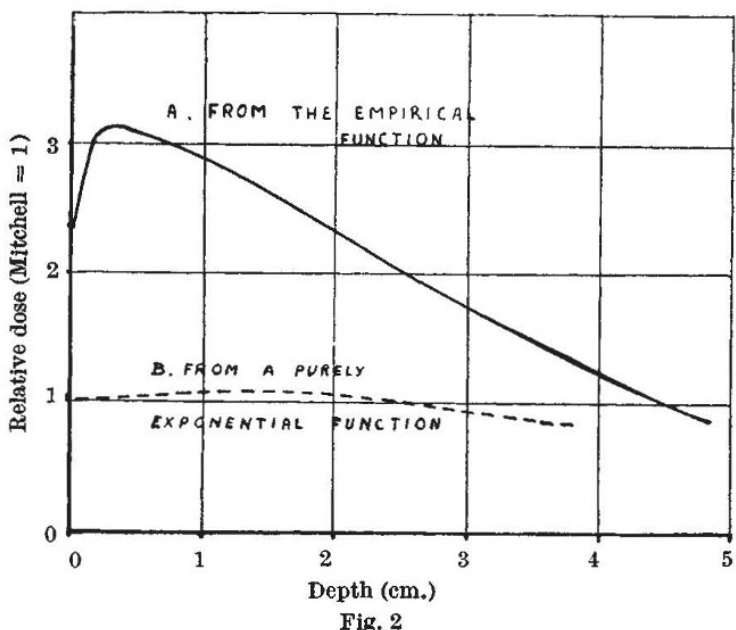

experimental arrangement, 65 per cent of outside and surface activities comes from neutrons travelling in the direction howitzer-paraffin. The neutron distribu. tion function is then given by:

$$
N=N_{0}\{2 \cdot 55 \exp (-x / L)-\exp (-x / l)\},
$$

for an incident flux of $N_{0}$ neutrons $/ \mathrm{cm} .^{2} / \mathrm{sec}$, and with $L=2.1 \mathrm{~cm}$. and $l=0.38 \mathrm{~cm}$., equal to the thermal neutron data as given by Fermi².

This result is the outcome of, on one hand, an inside diffusion of neutrons, and, on the other, near the surface there is a leakage up to a distance which is of the order of the mean free path.

We have worked out the ionization arising from such a function at the surface as well as in depth, and extended Mitchell's calculations in depth for a purely exponential function (full details will be published elsewhere); the results are as follow :

(1) For a given incident neutron flux the surface dose is double that worked out by Mitchell (Fig. 2).

(2) The ionization dose passes through a maximum at $0.3 \mathrm{~cm}$. with an intensity three times Mitchell's value.

To keep within the limits of the maximum tolerable dose, and taking into account the contribution of the $\mathrm{N}(n, p) \mathrm{C}$ reaction, we suggest that the maximum flux of incident neutrons should be limited to about 500 neutrons $/ \mathrm{cm}^{2} / \mathrm{sec}$.

\section{P. Capron \\ M. FAES \\ G. C. TAVERnIER}

Institut Interuniversitaire de Physique Nucléaire, Centre de Louvain. Nov. 21.

1 Mitchell, Brit. J. Rad. (Feb. 1947).

${ }^{2}$ Fermi and Amaldf, Phys. Rev., 50, 899 (1936).

\section{Method of Steepest Descents in X-Ray Analysis}

IN the method of steepest descents, atomic co-ordinates are derived by minimizing a function of the observed and calculated structure amplitudes. Hughes ${ }^{1}$ minimizes $\Sigma w\left(F_{0}-F_{c}\right)^{2}$, where the weighting factors are $w \sim 1 / F_{0}^{2}$ when $F_{0}>4 F_{t}$, and $w \sim 1 / 16 F_{t}^{2}$ when $F_{0}<4 F_{t} ; F_{t}$ is the threshold value of the measurement. As the errors do not obey Gauss' law, this formula does not give the best least-squares solution. 